Revista Brasileira de Fisiologia do exercício 2018;17(3):138-40

EDITORIAL

Atividade física: da saúde a performance

Antônio Carlos Leal Cortez

Centro Universitário Santo Agostinho UNIFSA, Teresina/PI, Programa de Pós-Graduação Stricto Sensu em Enfermagem e Biociências (PPgEnfBio), Doutorado Universidade Federal do Estado do Rio de Janeiro (UNIRIO), RJ, Bolsista Demanda Social CAPES, Pesquisador do Laboratório de Biociências da Motricidade Humana (LABIMH - UNIRIO), Fisiologista da Confederação Brasileira de Atletismo (CBAt)

\title{
Correspondência: antoniocarloscortez@hotmail.com
}

É notório que a prática regular da atividade física, de forma geral, proporciona vários benefícios à saúde e ainda constitui uma forma efetiva como prevenção de doenças e na promoção da saúde. Todavia, estudos epidemiológicos, Shibata et al. [1] e HHS [2], apontam que o século XXI está sendo marcado pelo crescimento progressivo da inatividade física, tanto em países desenvolvidos como em países em desenvolvimento, como é o caso do Brasil.

Segundo evidências científicas do American College of Sports Medicine (ACSM) [3], ACSM [4], Pitanga \& Lessa [5], níveis de atividade física abaixo do estabelecido, de acordo com as diretrizes internacionais vigentes ACSM [6], Advisory Committee on International Physical Activity Questionnaire [7], Institute of Medicine [8], Organização Mundial da Saúde (OMS) [9] e União Europeia [10], estão associadas à incidência de doenças relacionadas com o estilo de vida como síndrome metabólica, doenças crônicas não-transmissíveis (DCNT), marcadores inflamatórios e câncer.

Ressalta-se que a preocupação com o nível de atividade física vem desde a década de 1950, seja nos esportes de rendimento (voltados para a obtenção de resultados de aptidão física) bem como seus benefícios a saúde geral do indivíduo [2]. Entretanto, estudos como os de Mitchell et al. [11], Brawner, Churilla Júnior, Keteyian [12], Ferreira et al. [13] apontam que ainda é observado uma alta prevalência de inatividade física, entre diferentes populações no Brasil e no mundo, mesmo com a prática regular de atividade física apontada como um fator primordial na promoção da saúde e prevenção de algumas patologias [14-17].

Dentro desse contexto, faz-se importante diferenciar conceitualmente atividade física de exercício físico. Segundo Nahas [18], atividade física é todo movimento corporal que leva a contração de músculos estriados esqueléticos, levando o corpo a gastos energéticos acima dos níveis de repouso. Por sua vez, exercício físico é todo e qualquer movimento corporal, que mantém ou melhora a aptidão física, capacidade funcional e/ou outros componentes relacionados às valências físicas, como melhora na composição corporal, aumento da flexibilidade, aperfeiçoamento das habilidades atléticas, aumento da capacidade cardiorrespiratória, além de benefícios psicossociais como aumento da autoestima, aceitação social e sensação de bem-estar [19-21].

Entretanto, faz-se importante ressaltar que os objetivos planejados dentro de um programa de atividades físicas e/ou exercícios físicos sejam alcançados com a máxima eficiência e eficácia. Recomenda-se que todo processo desde avaliação, prescrição e acompanhamento sejam realizados por um profissional de Educação Física qualificado, devidamente credenciado ao Conselho - CREF/CONFEF. Dessa forma, estudos relacionados aos benefícios da intervenção de atividades e exercícios físicos orientados são de grande relevância, além de que tal temática não se esgota no mundo contemporâneo, em virtude do seu estado dinâmico [22]. 
1. Shibata A, Oka K, Nakamura Y, Muraoka I. Prevalence and demographic correlates of meeting the physical activity recommendation among Japanese adults. J Phys Act Health 2009;6(1):24-32.

2. HHS. U.S. Department of Health and Human Services. Physical activity guidelines advisory committee report. Washington: U.S. Department of Health and Human Services; 2008.

3. American College of Sports Medicine (ACSM). Appropriate physical activity intervention strategies for weight loss and prevention of weight regain for adults. Med Sci Sports Exerc 2009;41(2):459-71.

4. American College of Sports Medicine (ACSM); American Diabetes Association (ADA). Exercise and Type 2 Diabetes: American College of Sports Medicine and the American Diabetes Association: Joint Position Statement. Med Sci Sports Exerc 2010;42(12):2282-2303.

5. Pitanga FJ, Lessa I. Relationship between leisure-time physical activity and blood pressure in adults. Arq Bras Cardiol 2010;95(4):480-4.

6. American College of Sports Medicine (ACSM). ACSM's health-related physical fitness assessment manual. 2nd ed. Philadelphia: Lippincott Williams \& Wilkins; 2007.

7. International Physical Activity Questionnaire (IPAQ). Guidelines for data processing and analysis of the International Physical Activity Questionnaire (IPAQ) — short and long forms. 2005. [citado 2018 Ago 21]. Disponível em: http://www.ipaq. ki.se/scoring.pdf

8. Institute of Medicine (IOM). Dietary reference intakes for energy, carbohydrate, fiber, fat, fatty acids, cholesterol, protein, and amino acids. Washington: National Academies Press. 2004. [citado 2018 Ago 22]. Disponível em: http://www.iedar. es/pdf/informes/cancer/Dietary-referenceintakes-energy-carbohydrate-fibe-fatfattyacids-cholesterol.pdf

9. Organização Mundial de Saúde (OMS). Global recommendations on physical activity for health. Geneva: WHO; 2010. [citado 2018 Set 2]. Disponível em: https://www.cabdirect.org/cabdirect/abstract/20133026906

10. União Europeia: Instituto do Desporto de Portugal. Orientações da União Europeia para a actividade física: Políticas recomendadas para a promoção da saúde e do bem-estar. 2008. [citado 2018 Set 2]. Disponível em: http://www.idesporto.pt/ficheiros/File/Livro_IDPfinalJan09.pdf

11. Mitchell JA, Dowda M, Pate RR, Kordas K, Froberg K, Sar-Dinha LB et al. Physical activity and pediatric obesity: a quantile regression analysis. Med Sci Sports Exerc 2017;49(3):466-73.

12. Brawner CA, Churilla Júnior, Keteyian SJ. Prevalence of physical activity is lower among individuals with chronic disease. Med Sci Sports Exerc 2016;48(6):1062-7.

13. Ferreira RW, Varela AR, Monteiro LZ, Häfele CA, Santos SJD, Wendt A et al. Desigualdades sociodemográficas na prática de atividade física de lazer e deslocamento ativo para a escola em adolescentes: Pesquisa Nacional de Saúde do Escolar (PeNSE 2009, 2012 e 2015). Cad Saúde Pública 2018;34(4).e00037917. http://dx.doi.org/10.1590/0102-311x00037917.

14. Silva PVC, Costa Júnior AL. Efeitos da atividade física para a saúde de crianças e adolescentes. Psicologia Argumento 2017;29(64).

15. Freitas V, Melo CCD, Leopoldino A, Boletini T, Noce F. Influência do nível de atividade física e da mobilidade sobre o estresse emocional em idosos comunitários. Revista de Psicología del Deporte 2018;27(4):75-81.

16. Matsuo RF, Velardi M, Camara FM, Miranda MLDJ. Saúde e doença: representações de mulheres idosas praticantes de atividade física. Revista de Psicología del Deporte 2018;27(4):97-102.

17. Pitanga FJG, Matos SM, Conceição Almeida M, Barreto SM, Aquino EM. Atividade física no tempo livre, porém não atividade física no deslocamento, está associada com risco cardiovascular em participantes do ELSA-Brasil. Arq Bras Cardiol 2018;110(1):3643.

18. Nahas MV. Atividade física, saúde e qualidade de vida: conceitos e sugestões para um estilo de vida ativo. 4. ed. Londrina: Midiograf; 2006. 
19. Stampfer M, Hu F, Manson J, Rimm E, Willett W. Primary prevention of coronary heart disease in women through diet and lifestyle. New England J Med 2000;343(1):16-23.

20. Antunes JMM, Ferreira RME, Moreira-Gonçalves D. Exercise training as therapy for cancer-induced cardiac cachexia. Trends in Molecular Medicine 2018;24(8):709-27.

21. Hu FB, Manson JE, Stampfer MJ, Colditz G, Liu S, Solomon CG, et al. Diet, lifestyle, and the risk of type 2 diabetes mellitus in women. New England $\mathrm{J}$ Med $2001 ; 345(11): 790-7$.

22. Conselho Federal de Educação Física - CONFEF. Documento de intervenção profissional de educação física nº 36, junho de 2010. 